\title{
Redox crosstalk at endoplasmic reticulum (ER) membrane contact sites (MCS) uses toxic waste to deliver messages
}

\author{
Edgar Djaha Yoboue ${ }^{1}$, Roberto Sitia ${ }^{1}$ and Thomas Simmen ${ }^{2}$
}

\begin{abstract}
Many cellular redox reactions housed within mitochondria, peroxisomes and the endoplasmic reticulum (ER) generate hydrogen peroxide $\left(\mathrm{H}_{2} \mathrm{O}_{2}\right)$ and other reactive oxygen species (ROS). The contribution of each organelle to the total cellular ROS production is considerable, but varies between cell types and also over time. Redox-regulatory enzymes are thought to assemble at a "redox triangle" formed by mitochondria, peroxisomes and the ER, assembling "redoxosomes" that sense ROS accumulations and redox imbalances. The redoxosome enzymes use ROS, potentially toxic by-products made by some redoxosome members themselves, to transmit inter-compartmental signals via chemical modifications of downstream proteins and lipids. Interestingly, important components of the redoxosome are ER chaperones and oxidoreductases, identifying ER oxidative protein folding as a key ROS producer and controller of the tri-organellar membrane contact sites (MCS) formed at the redox triangle. At these MCS, ROS accumulations could directly facilitate inter-organellar signal transmission, using ROS transporters. In addition, ROS influence the flux of $\mathrm{Ca}^{2+}$ ions, since many $\mathrm{Ca}^{2+}$ handling proteins, including inositol 1,4,5 trisphosphate receptors (IP $\left.{ }_{3} \mathrm{Rs}\right)$, SERCA pumps or regulators of the mitochondrial $\mathrm{Ca}^{2+}$ uniporter (MCU) are redox-sensitive. Fine-tuning of these redox and ion signaling pathways might be difficult in older organisms, suggesting a dysfunctional redox triangle may accompany the aging process.
\end{abstract}

\section{Facts}

- The ER forms membrane contact sites (MCS) with mitochondria (mitochondria-associated membrane, MAM), as well as peroxisomes

- Redox-sensitive proteins localize to physical contacts between the endoplasmic reticulum (ER) and mitochondria, known as mitochondria-ER contacts (MERCs)

- Redox signaling takes place at the "redox triangle" formed by mitochondria, peroxisomes and the ER

Correspondence: Roberto Sitia (sitia.roberto@hsr.it) or Thomas Simmen (Thomas.Simmen@ualberta.ca)

${ }^{1}$ Protein Transport and Secretion Unit, Division of Genetics and Cell Biology, IRCCS Ospedale San Raffaele, Università Vita-Salute San Raffaele, Milan, Italy ${ }^{2}$ Department of Cell Biology, University of Alberta, Edmonton, $A B$,

CanadaT6G2H7

Edited by P. Pinton
- Given the hormetic properties of reactive oxygen species (ROS, essential or toxic, depending on their concentrations) their trans-organellar transport and diffusion are likely regulated

\section{Open Questions}

- Does a multimeric, mitochondria, peroxisome and ER-associated protein complex exist that forms a "redoxosome" whose composition differs from cell to cell?

- Does protein folding and/or the unfolded protein response (UPR) influence the redox state of mitochondria and peroxisomes, as well as their $\mathrm{Ca}^{2+}$ handling machinery?

- Are there ROS conducting channels at MCS? 
- What are the consequences of a dysfunctional redoxosome during aging?

\section{Introduction}

Reduction and oxidation reactions, summarized as redox, are reactions where there is gain (reduction) or loss (oxidation) of electron(s) by atoms of the reacting species. This plethora of chemical reactions governs virtually all aspects of life. Our essay focuses on the redox interplay between three organelles that produce reactive oxygen species (ROS), mitochondria, peroxisomes, and the endoplasmic reticulum $(E R)^{1}$. A common redox background of these organelles is reflected on the cell biological level by physical and functional contacts between the three that defy the classic textbook notion of stand-alone organelles with well-defined single functions ${ }^{2}$. These inter-organellar physical connections allow for the exchange of material in the form of metabolites, protein and lipid material, and ROS that influence the cellular redox $^{3}$. Although functional connections were initially hard to detect ${ }^{4}$, the first descriptions of organellar apposition between the three organelles were made during the early phases of electron microscopy studies in the mid 20th century, for example between the ER and mitochondria $^{5,6}$, or between the ER, mitochondria and peroxisomes ${ }^{7}$. Amongst these types of membrane contact sites (MCS), those between mitochondria and ER are probably the best studied. These contacts are biochemically known as mitochondria-associated membranes $(\mathrm{MAMs})^{8,9}$, where redox-regulatory proteins are enriched ${ }^{10,11}$.

Prominent MAM-localized regulators of ERmitochondria crosstalk are calnexin ${ }^{12,13}$, Ero1 $\alpha^{14,15}$ or selenon $/ \mathrm{SEPN} 1^{16}$. These chaperones and oxidoreductases, some of which produce ROS themselves, bind to $\mathrm{ER} \mathrm{Ca}^{2+}$ handling proteins and determine ER-mitochondria $\mathrm{Ca}^{2+}$ flux and, thus, mitochondrial metabolism via redoxdependent interactions ${ }^{17}$. Therefore, the mechanism of ER oxidative protein folding moonlights as a regulator of mitochondria metabolism ${ }^{18}$, as had been proposed in 1958 by Silvio and Anna Fiala who had discovered that the extent of mitochondrial respiration is proportional to the extent of ER protein production ${ }^{19}$.

However, the cellular array of ROS-dependent, redoxsensitive MCS is not limited to the MAM: While the ER may be the most significant ROS producer in some cell types, as assayed by fluorescent probes ${ }^{20}$, and mitochondria are well known producers of ROS via oxidative phosphorylation $^{21}$, peroxisomes are another source of $\operatorname{ROS}^{22}$ (summarized in Table 1). It is therefore expected that these organelles participate in redox exchanges with the ER and mitochondria. This is the case, for instance, during the metabolism of etherphospholipids that requires the transport of lipid intermediates from peroxisomes to the ER, where they undergo enzymatic reduction $^{23}$. In the case of mitochondria and peroxisomes, redox-controlling relationships occur for instance during beta-oxidation of fatty acids. While mitochondrial betaoxidation produces $\mathrm{CO}_{2}$ and $\mathrm{H}_{2} \mathrm{O}$ as end products ${ }^{24}$, peroxisomal beta-oxidation of fatty acids is incomplete and produces nicotinamide adenine dinucleotide (NADH) that must be shuttled to mitochondria for complete oxidation ${ }^{1}$. Conversely, NAD + is likely shuttled into peroxisomes by SLC25A17 in mammalian cells ${ }^{25}$. A related pathway can use the yeast peroxisomal matrix protein Mdh3p, which converts oxaloacetate to malate through the oxidation of $\mathrm{NADH}^{26}$, a reaction that is critical for the maintenance of the peroxisomal redox balance ${ }^{27}$. In mammalian cells, a similar shuttle system uses a peroxisomal lactate dehydrogenase that converts pyruvate to lactate, where SLC16A1 shuttles pyruvate in and lactate out $^{28}$. At the moment, it is unclear whether similar shuttling is involved in the metabolism of very long chain fatty acid (VLCFA) degradation products from peroxisomes to mitochondria ${ }^{29}$.

Although all three organelles are equipped with redoxpreserving defense mechanisms, ROS are thought to rapidly diffuse across membranes via certain aquaporins or other specific proteinaceous channels leading to fateful accumulations of these signaling molecules in the proximity of the three organelles ${ }^{30,31}$, as well as potentially lipid droplets $^{32}$. From here, they may influence organellar homeostasis on the redox triangle: for instance, the inhibition of peroxisomal catalase, concomitant with increasing ROS, results in mitochondrial redox imbalance $^{33}$. Moreover, peroxisomal catalase is under the control of the ER unfolded protein transcription factor Xbp-1, suggesting a need for this enzyme during ER stress $^{34}$.

Thus, we propose that ternary ER-mitochondriaperoxisome structures form a "redox triangle", mediated by tethering complexes between the ER and mitochondria $^{35}$, between peroxisomes and mitochondria ${ }^{36}$, as well as between peroxisomes and the $\mathrm{ER}^{37,38}$ (summarized in Fig. 1). The redox triangle may become dysfunctional with aging, as shown by decreases of peroxisomal catalase in older cells ${ }^{39}$.

\section{ROS sources and sinks in mitochondria, peroxisomes and the ER}

The powerful oxidant dioxygen or molecular oxygen $\left(\mathrm{O}_{2}\right)$ exhibits a particular chemistry ${ }^{40,41}$, also termed the molecular $\mathrm{O}_{2}$ paradox ${ }^{42}$. While $\mathrm{O}_{2}$ reduction ultimately can result in its transformation to water, this reduction requires the acceptance of one electron at a time. The $\mathrm{O}_{2}$ chemical behavior frequently results in incomplete $\mathrm{O}_{2}$ reductions, generating oxidizing species which can (non) catalytically react with biomolecules. Such oxidizing 


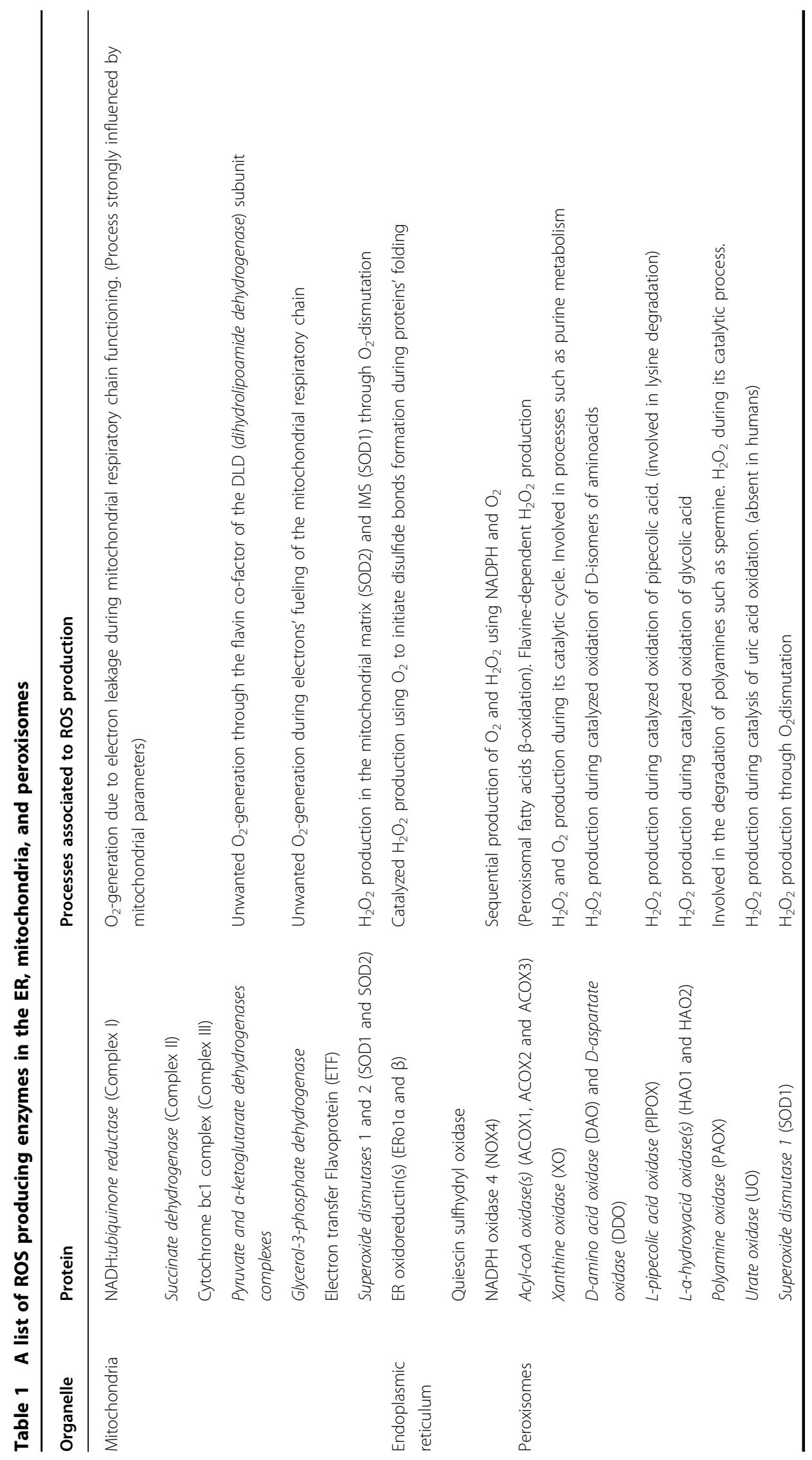




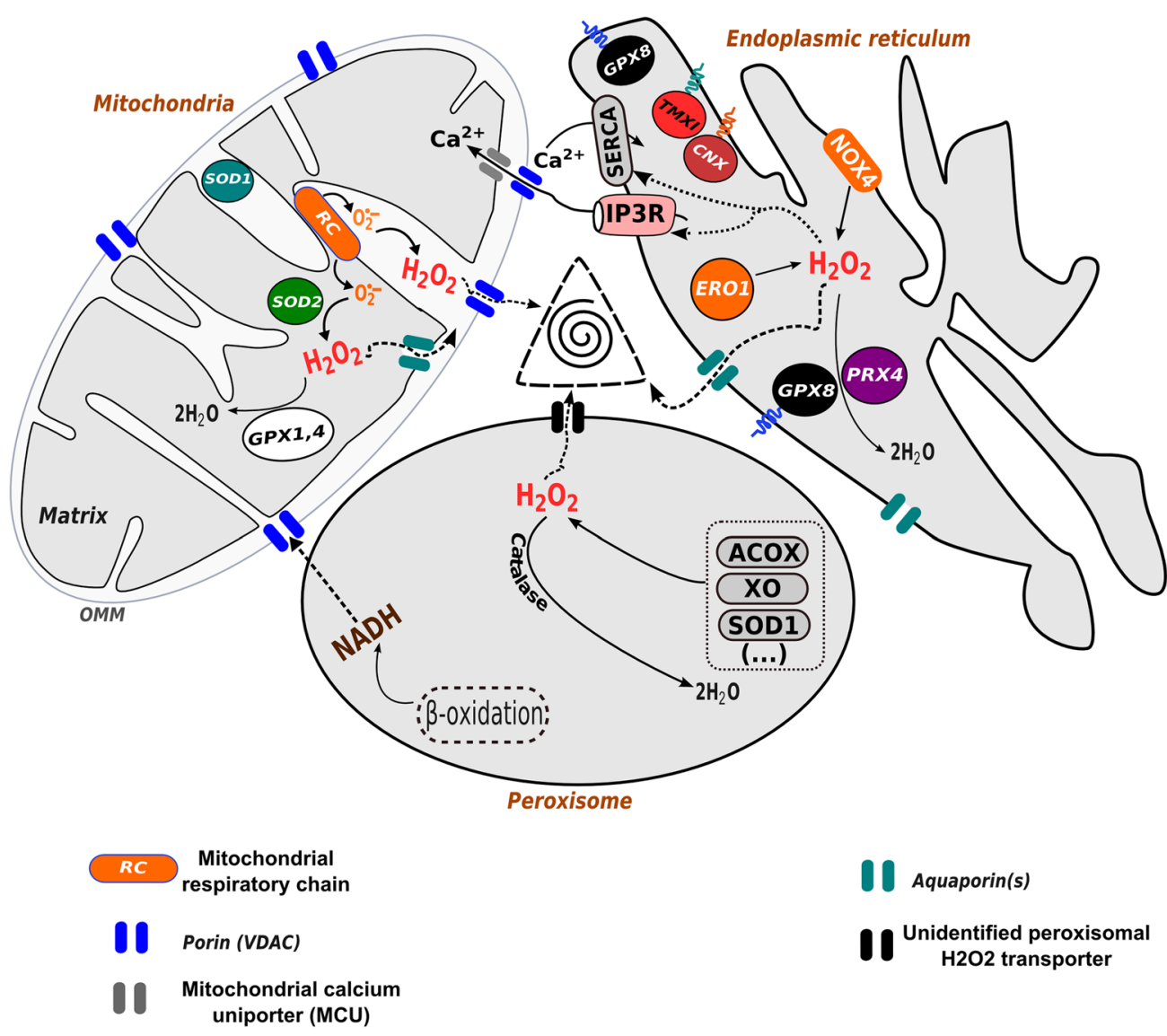

Fig. 1 The ER-mitochondria-peroxisome redox triangle. The endoplasmic reticulum (ER), mitochondria and peroxisomes are three important redox-sensitive organelles. All three house biochemical reactions that produce reactive oxygen species (ROS), for the list of ROS producers see Table 1. ROS can be released by all three organelles through aquaporins or as of yet unknown proteinaceous channels. Accumulated ROS within the redox triangle affect the functioning of ER-mitochondria $\mathrm{Ca}^{2+}$ exchange, oxidative phosphorylation, and especially oxidative protein folding within the ER

species are ROS, like superoxide anion, hydroxyl radicals, hypochlorous acid and hydrogen peroxide $\left(\mathrm{H}_{2} \mathrm{O}_{2}\right)$ to name but a few examples.

While basal ROS levels by mitochondria, peroxisomes and the ER are low due to organellar defense systems, redox modifications especially on the mitochondrial respiratory chain can strongly impact on ROS production and can trigger mitochondrial-induced oxidative stress $^{43,44}$. As outlined by ref. 45 , the organellar ROS production is dependent on cell and tissue types, as well as oxygen tension. Considering absolute production within the three organelles and their relative permeability for ROS (Table 1), the ER emerges as the relative most important contributor to cytosolic ROS amounts from purified organelles with approximately $60 \%$ of ROS derived from this organelle, and the remainder evenly split between mitochondria and peroxisomes ${ }^{45}$. Due to the diverse amounts of ROS scavengers within the three organelles (Table 2), the absolute ROS production amounts are, however, different. When examining perfused liver tissue, the contribution of the ER to total ROS production is negligible, while peroxisomes emerge as a prime ROS production site. In addition to diverse levels of ROS scavengers found within the peroxisomes, mitochondria and the ER, these observations also suggest that the proper formation of contacts between the ER, mitochondria, and peroxisomes could determine the relative cellular ROS contribution of each organelle. Altered cell growth conditions, such as altered protein folding demand, may impact on these findings.

The most important source of the high amounts of ROS within peroxisomes are the enzymatic activities of oxidases $^{46}$. Amongst these are fatty acid beta-oxidation by acyl-CoA oxidases (ACoX), a group of flavin adenine dinucleotide (FAD)-dependent enzymes under the control of peroxisome-proliferator activated receptors (PPARs) that oxidize long acyl-CoAs ${ }^{47}$. Peroxisomes are equipped with their own defense system that comprises catalase, 


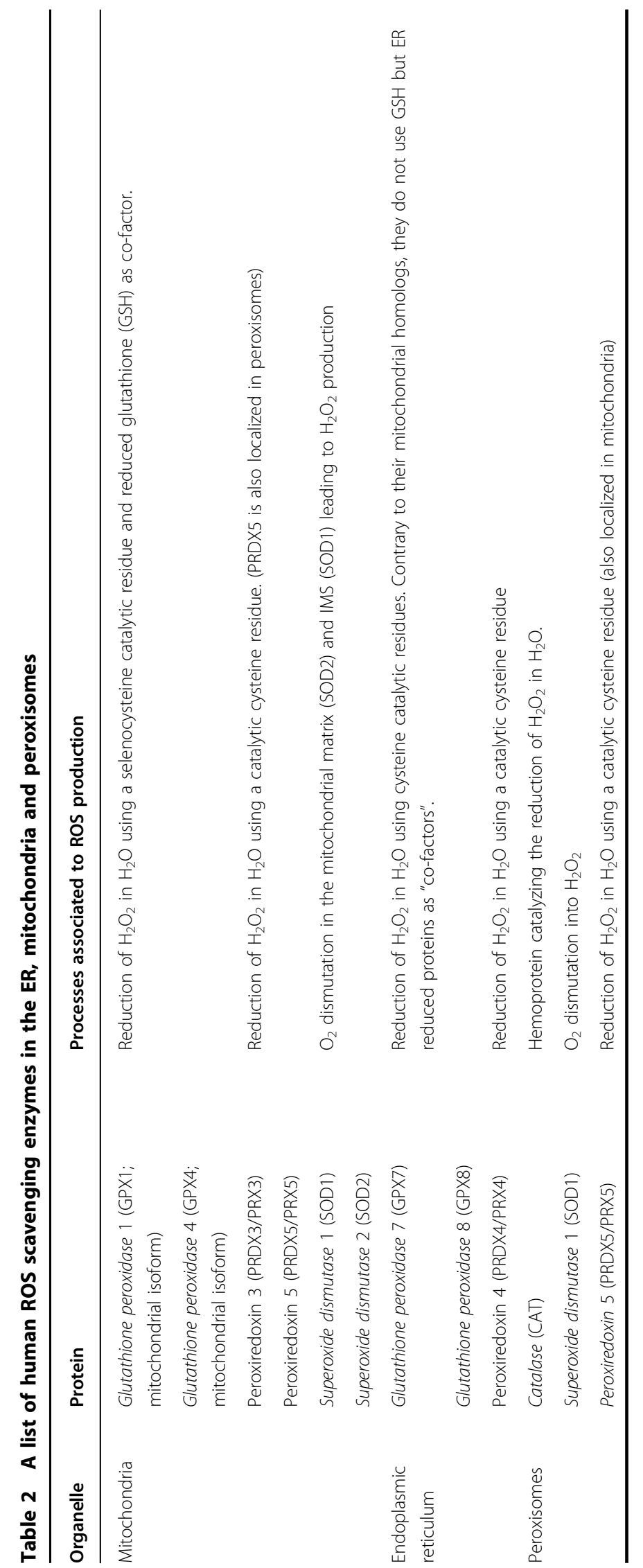




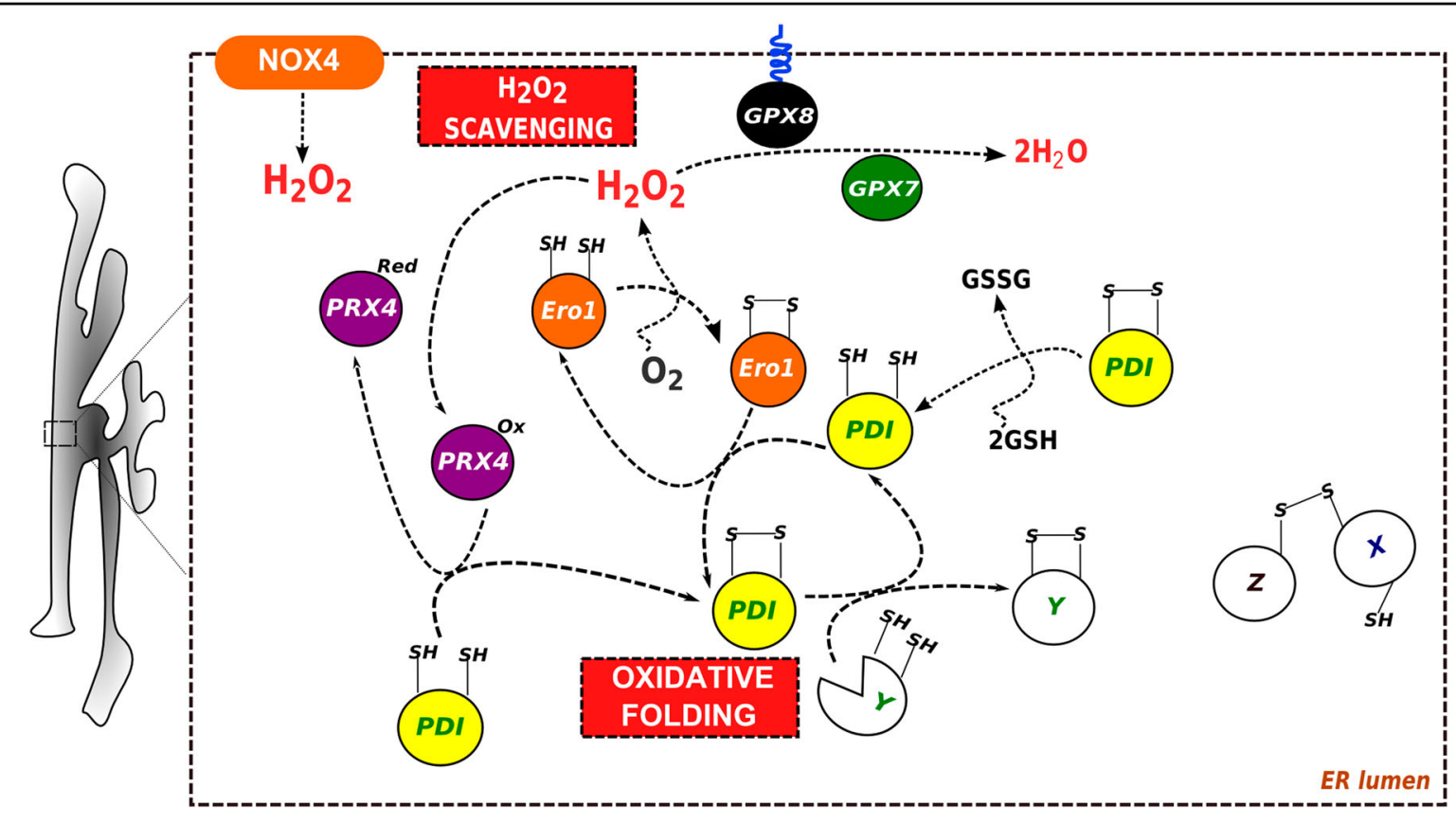

Fig. 2 Overview of the ER redox processes. The oxidation of Erol by $\mathrm{O}_{2}$ initiates disulfide relays leading to the insertion of disulfide bonds into proteins as they fold in the ER (here, the protein " $Y$ ") with oxidoreductases of the PDI family playing a key intermediary role (see main text for details). $\mathrm{H}_{2} \mathrm{O}_{2}$ produced during Erol oxidation can be scavenged by the peroxidases Gpx7, Gpx8 and Prx4. $\mathrm{H}_{2} \mathrm{O}_{2}$ is also formed in the ER NOX4. Upon $\mathrm{H}_{2} \mathrm{O}_{2}$ scavenging, oxidized Prx4 and Gpx7 transfer a disulfide bond to PDI, contributing to oxidative protein folding. Intermolecular disulfide bonds are also formed into the ER for establishing oligomeric covalent structures or for example retaining misfolded proteins

SOD and Prx $5 / \mathrm{PMP} 20^{46}$, which absorb the majority of the organelle's ROS, resulting in only minor amounts of cytosolic ROS derived from peroxisomes ${ }^{45}$.

This is not the case for mitochondrial and ER ROS, although these organelles also have their ROS defense systems in the form of peroxiredoxins and other enzymes $^{48,49}$. The main ROS defense systems within mitochondria are superoxide dismutase 1 (SOD1) in the inter-membrane space (IMS) and SOD2 in the mitochondrial matrix. Both enzymes rapidly convert superoxide into $\mathrm{H}_{2} \mathrm{O}_{2}$. In addition, mitochondria also contain glutathione peroxidases, particularly associated to the outer mitochondrial membrane (OMM), where they protect lipids from oxidative modifications ${ }^{50,51}$. Another mitochondrial redox event is the catalyzed formation of disulfide bonds in the IMS. Indeed, contrary to the mitochondrial matrix where the aforementioned antioxidant systems restrict their formation, the yeast oxidoreductases Mia40 and Erv1 catalyze disulfide bonds in the IMS, thus forming a mitochondrial disulfide relay process $^{52}$. Here, $\mathrm{CHCHD} 4$, the human homolog of Mia40 interacts with apoptosis-inducing factor (AIF), a chaperone of oxidative phosphorylation ${ }^{53,54}$. This interaction determines $\mathrm{CHCHD} 4$ import to the IMS and, therefore, oxidative phosphorylation. The activity of Mia40/ $\mathrm{CHCDH} 4$ is directly connected to the cytosolic redox, since changes thereof that can be transmitted through VDAC determine its activity ${ }^{55}$.

Inside the ER, ROS are produced from catalytic processes by the oxidoreductase Ero1 (Fig. 2) ${ }^{56}$ and by at least one member of the NADPH oxidase (NOX) family ${ }^{57}$. Ero1 uses the oxidative power of molecular oxygen to initiate redox relays which ultimately lead to disulfide bond generation in newly synthesized, folded proteins ${ }^{58}$, thus identifying oxidative protein folding as a major cellular ROS source ${ }^{59}$. Important partners in this relay are the members of the protein disulfide isomerase (PDI) family, folding assistants with thioredoxin (Trx)-like domains that catalyze the formation or isomerization of inter- and intra-molecular disulfide bonds into ER proteins ${ }^{60,61}$. Reduced PDI, the product of disulfide bond formation, retains catalytic functions as an isomerase, but also as an enzyme needed to eliminate misfolded proteins from the $E R$ via its reducing power, potentially in collaboration with another ER oxidoreductase, ERdj $5^{62,63}$. ER peroxidases, such as peroxiredoxin 4 (Prx4), as well as the glutathione peroxidases GPx7 and GPx8 scavenge luminal $\mathrm{H}_{2} \mathrm{O}_{2}$ arising from Ero1 and NOX, and normally prevent 
$\mathrm{H}_{2} \mathrm{O}_{2}$ leakage from the $\mathrm{ER}^{49,64-66}$ (Fig. 2). As an alternative pathway, Prx4 can convert $\mathrm{H}_{2} \mathrm{O}_{2}$ to disulfide bonds on nascent polypeptides ${ }^{66}$. This role of ROS may also rely on a chemical oxidation of PDI family proteins ${ }^{67}$ and on increased levels of oxidized glutathione (GSSG) in the presence of $\operatorname{ROS}^{68}$. It is currently not known whether an accumulation of mitochondrial or peroxisomal ROS could similarly mediate the catalytic formation of disulfide bonds.

Persistent ER stress increases production of ROS at the $\mathrm{ER}^{69}$, suggesting that the accumulation of unfolded proteins leads to redox-amplified imbalances in the Ero1/PDI electron flow ${ }^{70}$. These may be counteracted by an influx of reduced glutathione $(\mathrm{GSH})^{71-73}$. In that context, it is important to note the role of the cytosolic redox environment, since the inhibition of cytosolic thioredoxin reductase 1 influences successful ER disulfide bond formation ${ }^{74}$. The recent advent of redox probes for the cytosol, and the organelles of the redox triangle $e^{75-77}$ will facilitate further investigation of these questions.

\section{Targets of oxidative stress in mitochondria, peroxisomes and the ER}

The presence of antioxidant enzymes within mitochondria (SOD), peroxisomes (catalase) and the ER (peroxiredoxin 4 and glutathione peroxidase 7 and 8) initially suggested that ROS are toxic byproducts ${ }^{78}$. Thus, they were thought to promote aging: consistent with this idea, the cytosol of $C$. elegans cells becomes more oxidizing with age, while the ER becomes more reducing ${ }^{79}$. However, it is now firmly established that $\mathrm{H}_{2} \mathrm{O}_{2}$ acts as a key second messenger in cell growth or differentiation. Accordingly, dedicated transporters shuttle $\mathrm{H}_{2} \mathrm{O}_{2}$ between organelles, like aquaporins on the ER and the plasma membrane $e^{80,81}$. For instance, aquaporin-8 localizes to the $\mathrm{ER}^{82,83}$ or mitochondria ${ }^{84}$. Moreover, peroxisomes contain Pex11, which shows homology to transient receptor potential (TRPM) ion channels and transports beta-oxidation metabolites ${ }^{85}$, but a bona fide peroxisomal channel for ROS is currently not known ${ }^{86,87}$.

Owing to their chemical properties, ROS can modify target proteins, changing their conformation or activity.

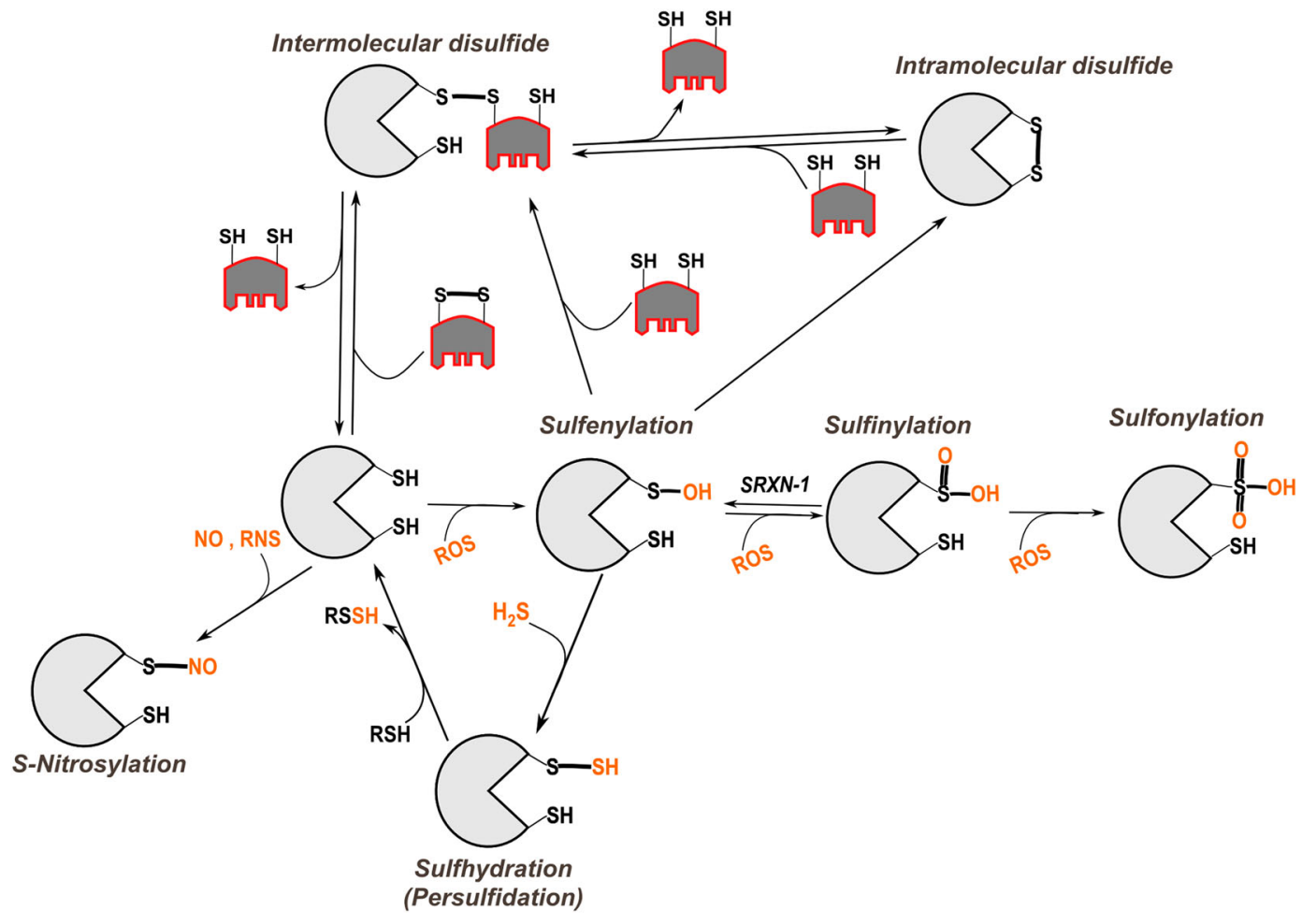

Fig. 3 Overview of redox post-translational modifications of cysteines. Oxidation by ROS (like $\mathrm{H}_{2} \mathrm{O}_{2}$ ) initially leads to sulfenylation ( $(\mathrm{SOH}$ ). Sulfenylated cysteine can additionally react with ROS leading first to sulfinylation $\left(\mathrm{SO}_{2} \mathrm{H}\right)$ and then to sulfonylation $\left(\mathrm{SO}_{3} \mathrm{H}\right)$. While sulfonylation is so far considered irreversible, sulfinylation can be reversed through the catalytic activity of the cytoplasmic enzyme sulfiredoxin-1 (SRXN-1). Many reactions can lead to disulfide bond formation: (i) intermolecular disulfide bonds can be formed with another protein or low molecular weight thiols (glutathione for example), (ii) intramolecular disulfide bonds are often inserted into a reduced protein by disulfide exchange (via formation of mixed disulfides) with GSSG or another oxidized protein (e.g., PDI) or through reaction of the relative instable sulfenylated cysteine. Please note that reactions involving thiol groups $(\mathrm{SH})$ implies the formation of thiolate $\left(\mathrm{S}^{\mathrm{S}}\right)$ through deprotonation and so are strongly dependent on the local pKa 
Reversible redox modifications provide a powerful signaling network, not unlike phosphorylation ${ }^{88}$. For instance, cysteine residues can adopt numerous oxidation states (SS, $\mathrm{SOH}, \mathrm{SO}_{2} \mathrm{H}, \mathrm{SO}_{3} \mathrm{H}, \mathrm{SNO}, \mathrm{SSH}$ etc.), offering a large panel of physiological protein redox modifications (Fig. 3) ${ }^{89,90}$.

ROS play important roles at the ER, where their production activates the unfolded protein response (UPR), accompanied by the inactivating sulfhydration of protein tyrosine phosphatase 1B (PTP1B) that results in the increased phosphorylation and, hence, activation of PERK $^{91}$. Another example is ROS-mediated sulfenylation, which shifts the activity of the UPR kinase Ire1 from ER stress signaling to activating $\mathrm{Nrf2}^{92}$. At the level of mitochondria, ROS lead to oxidation of components of the respiratory chain ${ }^{43}$ or sulfenylation of uncoupling protein-1, resulting in increased thermogenesis ${ }^{93}$. Within peroxisomes or in the cytoplasm, peroxisome-derived $\mathrm{H}_{2} \mathrm{O}_{2}$ oxidizes $\operatorname{Prx} 2^{94,95}$, which leads to the degradation of this ROS scavenger ${ }^{96}$.

Cysteine oxidation can also engender intra- or intermolecular disulfide bonds that stabilize or regulate the tertiary and quaternary structures of proteins. This is shown for example with the Nrf2 transcription factor, where $\mathrm{H}_{2} \mathrm{O}_{2}$ mediates the formation of an activating disulfide bond within its regulatory partner KEAP1 ${ }^{97,98}$. Enzymes mediating ER oxidative protein folding such as BiP/Grp78 are also targets of ROS modifications that serve to modulate their chaperoning function ${ }^{67,99}$.

Another group of redox-controlled modifications on cysteines are glutathionylations. ER proteins are frequent targets of this modification ${ }^{100}$, including many important regulators of the interactions with mitochondria like calnexin and SERCA $\mathrm{Ca}^{2+}$ pumps $^{101}$. This modification results in the activation of SERCA ${ }^{102}$. At the level of mitochondria, ROS can trigger glutathionylation of the pyruvate dehydrogenase complex, another source of mitochondrial ROS, which further increases oxidative stress $^{103}$. Moreover, mitofusins can undergo redoxdependent disulfide bond formation, leading to their dimerization and potentially glutathionylation, which promotes mitochondrial fusion aiming to mitigate oxidative stress-mediated damage on oxidative phosphorylation ${ }^{104}$. At the same time, mitochondrial $\mathrm{Ca}^{2+}$ uniporter glutathionylation promotes activation of mitochondrial $\mathrm{Ca}^{2+}$ import, resulting in the activation of oxidative phosphorylation, but also increasing apopto$\mathrm{sis}^{105}$. Counteracting these modifications is the mitochondrial protease Lon, which degrades ROS-modified mitochondrial proteins ${ }^{106}$. Interestingly, peroxisomes also contain their own Lon protease (pLon), suggesting similar mechanisms may be at play in this organelle as well ${ }^{107}$. This peroxisomal protease can interact with enzymes involved in beta-oxidation and mediate their activation ${ }^{108}$, but peroxisomal Lon also plays a role in the sorting of PTS1 proteins and can act as a chaperone ${ }^{109}$.

The role of ROS also has an inter-organellar dimension: for example, ROS leaking from mitochondria interact with glyceraldehyde 3-phosphate dehydrogenase (GAPDH), which inactivates the active cysteine of this enzyme via formation of a disulfide bond, and hence blocks glycolysis ${ }^{110}$. Moreover, mitochondrial ROS also dictate the cellular disulfide proteome ${ }^{111}$, suggesting that mitochondrial ROS cross-influence in particular the ER. Similarly, increased peroxisomal ROS production can leak to the cytosol, where it can oxidize important signaling molecules, including NF-kB and PTEN ${ }^{94}$. ROS leak could also occur across aquaporin-8, the ER ROS pore ${ }^{112}$. Together, these currently preliminary observations raise the possibility that redox signaling functions in interorganellar and intercellular ways between the "redox factories" of eukaryotic cells: mitochondria, peroxisomes and the ER.

\section{ER-mitochondria redox signaling via mitochondria-associated membranes (MAMs)}

The ER is a multitask organelle that coordinates the "validation" or degradation of secretory proteins, but also synthesizes lipids and is the main intracellular $\mathrm{Ca}^{2+}$ store with a luminal concentration close to the extracellular milieu $(0.5-1 \mathrm{mM})^{113,114}$. Since the mid 20th century, researchers had noticed that some ER regions were in close proximity with mitochondria ${ }^{5}$ and also that mitochondria isolated by classical subfractionation were tightly associated to ER tubules and vice versa ${ }^{115,116}$. In 1990, enzymes catalyzing the synthesis of phosphatidylserine (PS) and phosphatidylcholine (PC) were shown to localize to such ER-mitochondria contact sites, called mitochondria-associated membranes (MAMs) ${ }^{9}$. A combinatorial approach using light microscopy techniques and $\mathrm{Ca}^{2+}$ probes showed that mitochondria-ER contacts (MERCs) also use juxtapositions between $\mathrm{ER} \mathrm{Ca}^{2+}$ release channels and mitochondrial $\mathrm{Ca}^{2+}$ uniporter (MCU) to shuttle this ion from one organelle to the other ${ }^{117}$.

Given that mitochondria and ER are two intracellular redox hubs, it would come as no surprise if redox-related communication between the two occurred as well. Consistent with this hypothesis, the Hajnoczky lab recently managed to target a $\mathrm{H}_{2} \mathrm{O}_{2}$-specific fluorescent probe to $\mathrm{MAMs}^{31,118}$. Using this new tool, they detected the $\mathrm{Ca}^{2+}$ -dependent generation of redox nanodomains at the level of ER-mitochondria contact sites. These nanodomains were formed by mitochondrial $\mathrm{H}_{2} \mathrm{O}_{2}$ release upon $\mathrm{IP}_{3} \mathrm{R}$ mediated $\mathrm{Ca}^{2+}$ release at the interface between both organelles that caused $\mathrm{K}^{+}$and water influx into the mitochondrial matrix. The ensuing mitochondrial matrix swelling decreased the mitochondrial cristae volume, provoking the release of their $\mathrm{H}_{2} \mathrm{O}_{2}$ content. 
At this point, it is unclear what the consequences of this local ROS accumulation at the ER-mitochondria MCS are, but ryanodine receptors ${ }^{119}, \mathrm{IP}_{3} \mathrm{Rs}$ and SERCA $\mathrm{Ca}^{2+}$ pumps are known targets of ROS, which alter their activity $^{120}$. The readout of MAM-localized ROS is expected to result in SERCA inactivation ${ }^{121,122}$ and $\mathrm{IP}_{3} \mathrm{R}$ activation ${ }^{123}$, thus leading to a feed-forward loop for ERmitochondria $\mathrm{Ca}^{2+}$ flux. The ER oxidoreductases ERdj5 and SEPN1 counteract this accelerated ion flux, and activate SERCA by reducing its oxidation ${ }^{16,124}$. Conversely, ERp44 interacts with $\mathrm{IP}_{3} \mathrm{R} 1$ under reducing conditions to inhibit ER $\mathrm{Ca}^{2+}$ release ${ }^{125}$. Opposing these protective functions, other oxidoreductases act to boost ER-mitochondria $\mathrm{Ca}^{2+}$ crosstalk. Two prominent examples are Ero1 $\alpha$ and TMX1. Ero1 $\alpha$ acts on $\mathrm{IP}_{3}$ Rs, potentially competing with ERp $44^{15}$, increasing $\mathrm{Ca}^{2+}$ release from the ER. Moreover, Ero1 $\alpha$ reduces mitochondrial $\mathrm{Ca}^{2+}$ uptake ${ }^{14}$. Conversely, TMX1 inactivates SERCA ${ }^{126}$, thus reducing $\mathrm{Ca}^{2+}$ uptake from the cytosol. A more ambiguous role is played by GPx8, which inactivates SERCA, but due to the reduction of the $\mathrm{ER} \mathrm{Ca}^{2+}$ reservoir, also reduces ER-mitochondria $\mathrm{Ca}^{2+} \mathrm{flux}^{11}$. Through their combined activities, these oxidoreductases, previously considered exclusively part of the ER folding machinery, form a redoxosome at the MAM, where they predominantly localize $\mathrm{e}^{10,11,14,126}$. Such a redoxosome would be a multimeric, multiorganellar protein complex that mediates or controls MCS formation in a redox-specific manner. It is currently unclear whether any ROS production from Ero1 $\alpha$ itself influences the redoxosome from the MAM.

Bax inhibitor 1 (BI-1) localizes to MAM as well ${ }^{127}$ and reduces ER ROS in a heme oxygenase 1-dependent manner, suggesting the ER has redox-controls that go beyond the GPx proteins ${ }^{128}$. In addition, BI-1 has further roles for the ER redox due to its association with the NADPH-dependent cytochrome P450 reductase ${ }^{129}$.

Less is known about redox-controlled proteins that localize to the mitochondrial part of MCS. One example is p66Shc, which localizes to the IMS and partially to MAM, particularly in cells stressed from UV radiation or $\mathrm{H}_{2} \mathrm{O}_{2}{ }^{130,131}$. At the MAM, p66Shc stimulates ROS production via interference with oxidative phosphorylation, and influences apoptotic pathways ${ }^{132}$. This occurs following the phosphorylation by protein kinase $\mathrm{C}$ beta upon a shift of cellular redox to oxidizing conditions ${ }^{133}$. p66Shc is not only a thiol-reactive molecule ${ }^{134}$, but it also represses the expression of antioxidant proteins such as $\mathrm{SOD}^{135}$. Further connections between the IMS and MAM redox signaling are based on Mia40/CHCHD4, which can interact with MICU1 ${ }^{136}$. Upon interaction with Mia40, this key regulator of MCU forms a disulfide bond with its sister protein MICU2 and thus regulates $\mathrm{Ca}^{2+}$ uptake.
A potential link of MAM redox reactions exists with the machinery that mediates mitochondrial dynamics. GSSG promotes mitochondrial fusion, priming glutathionylation of mitochondrial mitofusins, in the case of mitofusin- 2 on cysteine $684^{137}$, thought to be located on the cytoplasmic portion of mitochondria ${ }^{138}$. While GSH causes the reduction of this cysteine and reduces electron flux on the oxidative phosphorylation chain ${ }^{104}$, ERderived ROS could have the opposite effect, thus outlining a pathway that could directly connect ER oxidative protein folding with mitochondrial dynamics ${ }^{139}$. In contrast, arrest of oxidative phosphorylation by hypoxia triggers the outer mitochondrial membrane protein FUNDC1 to exchange MAM-localized calnexin for Drp1 and thus, to mediate mitochondria fission ${ }^{140}$. Remarkably, these findings indicate that oxidative phosphorylation is monitored at the level of the MAM using ROS output by ER chaperones to elicit alterations in mitochondrial structure.

Redox imbalances in the ER have consequences inside mitochondria as well. This is nicely demonstrated by the mitochondrial protease Lon whose expression increases upon ER stress ${ }^{141}$ and an increase of mitochondrial respiration during ER stress ${ }^{142}$. Conversely, mitochondrial ROS exacerbate ER stress ${ }^{143}$, suggesting there is a feedback loop that reinforces ROS production in both organelles ${ }^{144}$. In yeast, this mechanism has recently been shown to depend on the ER-resident NADPH oxidase Ynolp that ramps up ER ROS production upon loss of cytochrome c oxidase. This finding led to the surprising identification of the ER as a prime source of ROS following problems at the level of oxidative phosphorylation ${ }^{145}$. Underscoring the importance of the ER as a ROS producer and sentinel for redox imbalances, the oxygensensing HIF1 $\alpha$ transcription factor localizes to the ER, where it undergoes a stabilizing Fenton reaction that depends on $\mathrm{pO}_{2}$ and subsequently enters the nucleus to trigger the hypoxia response ${ }^{146}$. At the moment, it is unclear whether these important redox communication pathways between the ER and mitochondria require a functional MAM.

\section{Peroxisomes contribute to the redoxosome}

Intriguingly, peroxisomes localize to triple contact sites with the ER and mitochondria in yeast ${ }^{147}$, formed via the interaction of Pex11 with the ER-mitochondria encounter structure (ERMES) ${ }^{148}$. These findings support the idea that they are part of the cellular redoxosome, forming a "redox triangle". Redox activities in peroxisomes influence mitochondrial redox ${ }^{33}$. Peroxisomes are connected to the ER and mitochondria in many ways: the connection to the ER is obvious, given the de novo biogenesis of peroxisomes originates at the $\mathrm{ER}^{149}$, especially when considering mammalian cells ${ }^{150}$, where ER structures can wrap 
around peroxisomes ${ }^{151,152}$. Both mitochondrial and ER structures are compromised in the absence of peroxisomal activity in a Pex5 knockout model ${ }^{153}$. These abnormalities include the swelling of mitochondria and increased ROS production by the oxidative phosphorylation electron relay ${ }^{154}$. In liver cells, peroxisome deficiency from a Pex2 knockout, likely resulting in increases of local ROS levels, leads to ER stress and PERK activation ${ }^{155}$. In contrast, plant peroxisomal catalase depends on $\mathrm{Ca}^{2+}$ import into the organelle ${ }^{156}$, which is tied to the availability of cytosolic (and therefore presumably ERreleased) $\mathrm{Ca}^{2+}{ }^{157}$.

Furthermore, the production of peroxisomal ROS can trigger mitochondrial apoptosis pathways, suggesting ROS are transmitted over to this organelle ${ }^{158}$. Indeed, ROS are thought to readily cross peroxisomal and mitochondrial membranes ${ }^{159,160}$. Such functional connections are not surprising, given that beta-oxidation of fatty acids can be initiated in peroxisomes, but must be concluded in mitochondria ${ }^{161}$. The shuttling of the beta-oxidation intermediates may occur at physical contacts between the two organelles ${ }^{162}$ or potentially via as of yet uncharacterized bi-directional vesicular trafficking ${ }^{163}$, likely involving carnitine acetyl transferases ${ }^{164}$. Interestingly, mitochondria and peroxisomes share key components of their division machinery ${ }^{165}$, raising the possibility for such a currently hypothetical mechanism.

The requirement to replenish $\mathrm{NAD}^{+}$needed for beta-oxidation also suggests the existence of additional redox shuttle systems between the peroxisome and mitochondria involving malate and aspartate in yeast ${ }^{166}$, but their identity is currently not known in mammalian cells ${ }^{1}$. At peroxisome-mitochondria MCS, further redox shuttles may promote the reduction of NADP to $\mathrm{NADPH}^{167}$. The redox-related link between peroxisomes and mitochondria is further illustrated by the improvement of mitochondrial functioning upon increased amounts of peroxisomal catalase or peroxisomal betaoxidation ${ }^{168,169}$. However, these effects may be connected to the simple increase of ROS, concomitant with an increased level of oxidative stress upon peroxisome interference ${ }^{154}$.

Interestingly, particularly mammalian mitochondria might communicate with peroxisomes in the inverse direction via the generation of mitochondrial preperoxisomes carrying peroxisomal proteins including Pex $3{ }^{170}$, Pex12, Pex13, to name but a few examples, that fuse with ER peroxisomal precursors ${ }^{171}$. At this point, it is not clear whether these structures mediate redox crosstalk or whether they are ROS-sensitive, although their existence opens up such a possibility. As mentioned earlier, it is also not clear whether an inverse trafficking mechanism exists.

\section{Conclusion and perspectives}

The 21st century has witnessed the molecular characterization of membrane contact sites (MCS) that regulate the intracellular spatial organization of the compartments in eukaryotic cells. However, we have likely only seen the tip of the iceberg in terms of their full functionality. Thus, much has to be learned about the molecular events that mediate MCS crosstalk and how it is regulated. The molecular targets of the tight MAM-ER redox and $\mathrm{Ca}^{2+}$ signaling interplays remain largely uncharacterized. Further integration of redox signaling will also have to take into account the seemingly equal importance of peroxisomes to form a "redox triangle" that could depend on a multiprotein complex we propose to call the MCS "redoxosome". The known dysfunctions of all three components of the cellular redox triangle formed by mitochondria, peroxisomes and the ER during aging could really all arise from a dysfunctional redoxosome. The existence of the redoxosome could allow the cell to balance the production and elimination of ROS within the ER, the mitochondria and peroxisomes and adjust it to growth and stress conditions. This makes sense, since the upstream needs and downstream interactions of the three organelles are tightly connected. In fact, it is today unthinkable to imagine the functioning of any of the three organelles as a single unit, without the interaction with their redox partners. The best examples to illustrate these links are the shuttling of beta-oxidation intermediates between peroxisomes and mitochondria and the induction of mitochondrial proteins following ER stress.

Future experiments will have to test our proposal. Such experiments could involve the interference with organelle tethering, for instance by knocking out tethers that we postulate would be required for the proper functioning of the redoxosome. At the moment, such approaches can lead to results, which are difficult to interpret, as shows the example of mitofusin- $2^{172,173}$. One reason for such difficulties could be that the interference with tethers will affect functional interactions at MCS, including the redoxosome.

Currently, the identity of tethers is being elucidated not just for the ER and mitochondria ${ }^{174}$, but also for peroxisomes: pioneering studies in yeast have used peroxisome inheritance to identify a Pex3p-Inp1p complex that attaches them to the $\mathrm{ER}^{175}$. Using more recent BioID approaches in mammalian cells, a complex between ERlocalized VAMP-associated proteins A and B (VAPA and VAPB) and with the peroxisomal membrane protein acylCoA binding domain containing 5 (ACBD5) was shown to act as a tether as well ${ }^{37,38}$. Less is known about peroxisome-mitochondria tethers, but a recent publication has characterized a complex between Pex11 and ERMES as a putative tether ${ }^{148}$. 
To solve these questions enhanced efforts will be required to characterize biochemically and anatomically the MCS proteomes in time and space ${ }^{176}$. The evidence presented in this review that MCS may critically depend on multiple organelles interacting with each other presents both an unexpected difficulty for this task, but also a chance for exciting discoveries in future.

\section{Acknowledgements}

We thank Peter Kim and Tiziana Anelli for critical reading of the manuscript. Funding in the Simmen lab has been provided by CIHR (MOP 133541) to TS and by Telethon (GGP15059), Associazione Italiana per la Ricerca sul Cancro (AIRC, IG 14559) Ministero della Salute (PE-2011-02352286) and Fondazione Cariplo (2015-0591) to RS.

\section{Conflict of interest}

The authors declare that they have no conflict of interest.

\section{Publisher's note}

Springer Nature remains neutral with regard to jurisdictional claims in published maps and institutional affiliations.

Received: 24 July 2017 Revised: 29 September 2017 Accepted: 3 October 2017

Published online: 28 February 2018

\section{References}

1. Wanders, R. J., Waterham, H. R. \& Ferdinandusse, S. Metabolic interplay between peroxisomes and other subcellular organelles including mitochondria and the endoplasmic reticulum. Front Cell Dev. Biol. 3, 83 (2015).

2. Shai, N., Schuldiner, M. \& Zalckvar, E. No peroxisome is an island-peroxisome contact sites. Biochim. Biophys. Acta 1863, 1061-1069 (2016).

3. Schrader, M., Godinho, L. F., Costello, J. L. \& Islinger, M. The different facets of organelle interplay - an overview of organelle interactions. Front Cell Dev. Biol. 3, 56 (2015).

4. Shio, H. \& Lazarow, P. B. Relationship between peroxisomes and endoplasmic reticulum investigated by combined catalase and glucose-6phosphatase cytochemistry. J. Histochem. Cytochem. 29, 1263-1272 (1981).

5. Bernhard, W., Haguenau, F., Gautier, A. \& Oberling, C. Submicroscopical structure of cytoplasmic basophils in the liver, pancreas and salivary gland; study of ultrafine slices by electron microscope. Z. Zellforsch. Mikrosk. Anat. 37, 281-300 (1952)

6. Bernhard, W. \& Rouiller, C. Close topographical relationship between mitochondria and ergastoplasm of liver cells in a definite phase of cellular activity. J. Biophys. Biochem. Cytol. 2(Suppl. 4), 73-78 (1956).

7. Bernhard, W. \& Rouiller, C. Microbodies and the problem of mitochondrial regeneration in liver cells. J. Biophys. Biochem. Cytol. 2(Suppl. 4), 355-360 (1956).

8. Rusinol, A. E., Cui, Z., Chen, M. H. \& Vance, J. E. A unique mitochondriaassociated membrane fraction from rat liver has a high capacity for lipid synthesis and contains pre-Golgi secretory proteins including nascent lipoproteins. J. Biol. Chem. 269, 27494-27502 (1994).

9. Vance, J. E. Phospholipid synthesis in a membrane fraction associated with mitochondria. J. Biol. Chem. 265, 7248-7256 (1990).

10. Gilady, S. Y. et al. Erolalpha requires oxidizing and normoxic conditions to localize to the mitochondria-associated membrane (MAM). Cell. Stress Chaperones 15, 619-629 (2010).

11. Yoboue, E. D., Rimessi, A., Anelli, T., Pinton, P. \& Sitia, R. Regulation of calcium fluxes by GPX8, a Type-ll transmembrane peroxidase enriched at the mitochondria-associated endoplasmic reticulum membrane. Antioxid. Redox. Signal. 27, 583-595 (2017).

12. Lynes, E. M. et al. Palmitoylation is the switch that assigns calnexin to quality control or ER Ca2+ signaling. J. Cell. Sci. 126(Pt. 17), 3893-3903 (2013).

13. Myhill, $\mathrm{N}$. et al. The subcellular distribution of calnexin is mediated by PACS-2. Mol. Biol. Cell. 19, 2777-2788 (2008).
14. Anelli, T. et al. Erolalpha regulates $\mathrm{Ca}(2+)$ fluxes at the endoplasmic reticulum-mitochondria interface (MAM). Antioxid. Redox. Signal. 16, 1077-1087 (2012).

15. Li, G. et al. Role of ERO1-alpha-mediated stimulation of inositol 1,45-triphosphate receptor activity in endoplasmic reticulum stress-induced apoptosis. J. Cell. Biol. 186, 783-792 (2009).

16. Marino, M. et al. SEPN1, an endoplasmic reticulum-localized selenoprotein linked to skeletal muscle pathology, counteracts hyperoxidation by means of redox-regulating SERCA2 pump activity. Hum. Mol. Genet. 24, 1843-1855 (2015)

17. Gutierrez, T., Simmen, T. Endoplasmic reticulum chaperones tweak the mitochondrial calcium rheostat to control metabolism and cell death. Cell Calcium; $\quad$ pii: $\quad$ S0143-4160(17)30079-9; $\quad$ https:/doi.org/10.1016/j. ceca.2017.05.015 (2017).

18. Simmen, T., Lynes, E. M., Gesson, K. \& Thomas, G. Oxidative protein folding in the endoplasmic reticulum: tight links to the mitochondria-associated membrane (MAM). Biochim. Biophys. Acta 1798, 1465-1473 (2010).

19. Fiala, S. \& Fiala, A. E. On the correlation between metabolic and structura changes during carcinogenesis in rat liver. Br. J. Cancer 13, 136-151 (1959)

20. Malinouski, M., Zhou, Y., Belousov, V. V., Hatfield, D. L. \& Gladyshev, V. N. Hydrogen peroxide probes directed to different cellular compartments. PLoS ONE 6, e14564 (2011)

21. Murphy, M. P. How mitochondria produce reactive oxygen species. Biochem. J. 417, 1-13 (2009)

22. Sandalio, L. M., Rodriguez-Serrano, M., Romero-Puertas, M. C. \& del Rio, L. A. Role of peroxisomes as a source of reactive oxygen species (ROS) signaling molecules. Subcell. Biochem. 69, 231-255 (2013).

23. Das, A. K., Horie, S. \& Hajra, A. K. Biosynthesis of glycerolipid precursors in rat liver peroxisomes and their transport and conversion to phosphatidate in the endoplasmic reticulum. J. Biol. Chem. 267, 9724-9730 (1992).

24. Eaton, S., Bartlett, K. \& Pourfarzam, M. Mammalian mitochondrial betaoxidation. Biochem. J. 320(Pt. 2), 345-357 (1996).

25. Agrimi, G., Russo, A., Scarcia, P. \& Palmieri, F. The human gene SLC25A17 encodes a peroxisomal transporter of coenzyme A, FAD and NAD+. Biochem. J. 443, 241-247 (2012).

26. van Roermund, C. W., Elgersma, Y., Singh, N., Wanders, R. J. \& Tabak, H. F. The membrane of peroxisomes in Saccharomyces cerevisiae is impermeable to $\mathrm{NAD}(\mathrm{H})$ and acetyl-CoA under in vivo conditions. EMBO J. 14, 3480-3486 (1995).

27. Al-Saryi, N. A. et al. Two NAD-linked redox shuttles maintain the peroxisomal redox balance in Saccharomyces cerevisiae. Sci. Rep. 7, 11868 (2017).

28. McClelland, G. B., Khanna, S., Gonzalez, G. F., Butz, C. E. \& Brooks, G. A. Peroxisomal membrane monocarboxylate transporters: evidence for a redox shuttle system? Biochem. Biophys. Res. Commun. 304, 130-135 (2003).

29. Wanders, R. J. Metabolic functions of peroxisomes in health and disease. Biochimie. 98, 36-44 (2014).

30. Appenzeller-Herzog, $\mathrm{C}$. et al. Transit of $\mathrm{H}_{2} \mathrm{O}_{2}$ across the endoplasmic reticulum membrane is not sluggish. Free Radic. Biol. Med. 94, 157-160 (2016)

31. Booth, D. M., Enyedi, B., Geiszt, M., Varnai, P. \& Hajnoczky, G. Redox nanodomains are induced by and control calcium signaling at the ER-mitochondrial interface. Mol. Cell 63, 240-248 (2016).

32. Schuldiner, M. \& Bohnert, M. A different kind of love-lipid droplet contact sites. Biochim. Biophys. Acta 1862(10 Pt. B), 1188-1196 (2017).

33. Ivashchenko, O. et al. Intraperoxisomal redox balance in mammalian cells: oxidative stress and interorganellar cross-talk. Mol. Biol. Cell 22, 1440-1451 (2011).

34. Liu, Y. et al. Preventing oxidative stress: a new role for XBP1. Cell Death Differ. 16, 847-857 (2009).

35. De Vos, K. J. et al. VAPB interacts with the mitochondrial protein PTPIP51 to regulate calcium homeostasis. Hum. Mol. Genet. 21, 1299-1311 (2012).

36. Fan, J., Li, X., Issop, L., Culty, M. \& Papadopoulos, V. ACBD2/ECI2-mediated peroxisome-mitochondria interactions in Leydig cell steroid biosynthesis. Mol. Endocrinol. 30, 763-782 (2016).

37. Costello, J. L. et al. ACBD5 and VAPB mediate membrane associations between peroxisomes and the ER. J. Cell. Biol. 216, 331-342 (2017).

38. Hua, R. et al. VAPs and ACBD5 tether peroxisomes to the ER for peroxisome maintenance and lipid homeostasis. J. Cell. Biol. 216, 367-377 (2017).

39. Wood, C. S. et al. Hypocatalasemic fibroblasts accumulate hydrogen peroxide and display age-associated pathologies. Traffic 7, 97-107 (2006). 
40. Halliwell, B. Free radicals and antioxidants: updating a personal view. Nutr. Rev. 70, 257-265 (2012)

41. Winterbourn, C. C. Reconciling the chemistry and biology of reactive oxygen species. Nat. Chem. Biol. 4, 278-286 (2008).

42. Kuksal, N., Chalker, J. \& Mailloux, R. J. Review. Progress in understanding the molecular oxygen paradox - function of mitochondrial reactive oxygen species in cell signaling. Biol. Chem. 398, 1209-1227 (2017).

43. Bak, D. W. \& Weerapana, E. Cysteine-mediated redox signalling in the mitochondria. Mol. Biosyst. 11, 678-697 (2015).

44. Kowaltowski, A. J., de Souza-Pinto, N. C., Castilho, R. F. \& Vercesi, A. E. Mitochondria and reactive oxygen species. Free. Radic. Biol. Med. 47, 333-343 (2009)

45. CGiuliviABoverisECadenas2007The steady-state concentrations of oxygen radials in mitochondriaCColtonGGilbertReactive Oxygen Species in Biological Systems: an Interdisciplinary Approach.Kluwer Academic PublishersNew York76102Giulivi, C., Boveris, A. \& Cadenas, E. The steady-state concentrations of oxygen radials in mitochondria. In: C. Colton, G. Gilbert (eds). Reactive Oxygen Species in Biological Systems: an Interdisciplinary Approach. (pp. 76-102. Kluwer Academic Publishers, New York, 2007).

46. Antonenkov, V. D., Grunau, S., Ohlmeier, S. \& Hiltunen, J. K. Peroxisomes are oxidative organelles. Antioxid. Redox. Signal. 13, 525-537 (2010).

47. Van Veldhoven, P. P., Vanhove, G., Assselberghs, S., Eyssen, H. J. \& Mannaerts, G. P. Substrate specificities of rat liver peroxisomal acyl-CoA oxidases: palmitoyl-CoA oxidase (inducible acyl-CoA oxidase), pristanoyl-CoA oxidase (non-inducible acyl-CoA oxidase), and trihydroxycoprostanoyl-CoA oxidase. J. Biol. Chem. 267, 20065-20074 (1992).

48. Cox, A. G., Winterbourn, C. C. \& Hampton, M. B. Mitochondrial peroxiredoxin involvement in antioxidant defence and redox signalling. Biochem. J. 425 313-325 (2009).

49. Ramming, T. \& Appenzeller-Herzog, C. Destroy and exploit: catalyzed removal of hydroperoxides from the endoplasmic reticulum. Int. J. Cell. Biol. 2013, 180906 (2013).

50. Handy, D. E. et al. Glutathione peroxidase-1 regulates mitochondrial function to modulate redox-dependent cellular responses. J. Biol. Chem. 284, 11913-11921 (2009).

51. Ukai, Y., Kishimoto, T., Ohdate, T., Izawa, S. \& Inoue, Y. Glutathione peroxidase 2 in Saccharomyces cerevisiae is distributed in mitochondria and involved in sporulation. Biochem. Biophys. Res. Commun. 411, 580-585 (2011)

52. Erdogan, A. J. \& Riemer, J. Mitochondrial disulfide relay and its substrates: mechanisms in health and disease. Cell Tissue Res. 367, 59-72 (2017).

53. Hangen, E. et al. Interaction between AIF and $\mathrm{CHCHD} 4$ regulates respiratory chain biogenesis. Mol. Cell 58, 1001-1014 (2015).

54. Meyer, $\mathrm{K}$. et al. Loss of apoptosis-inducing factor critically affects MIA40 function. Cell Death Dis. 6, e1814 (2015).

55. Kojer, K. et al. Glutathione redox potential in the mitochondrial intermembrane space is linked to the cytosol and impacts the Mia40 redox state. EMBO J. 31, 3169-3182 (2012)

56. Ramming, T. et al. A PDI-catalyzed thiol-disulfide switch regulates the production of hydrogen peroxide by human Ero1. Free Radic. Biol. Med. 83, 361-372 (2015)

57. Laurindo, F. R., Araujo, T. L. \& Abrahao, T. B. Nox NADPH oxidases and the endoplasmic reticulum. Antioxid. Redox. Signal. 20, 2755-2775 (2014).

58. Tu, B. P. \& Weissman, J. S. The FAD- and O(2)-dependent reaction cycle of Ero1-mediated oxidative protein folding in the endoplasmic reticulum. Mol. Cell. 10, 983-994 (2002)

59. Hudson, D. A., Gannon, S. A. \& Thorpe, C. Oxidative protein folding: from thiol-disulfide exchange reactions to the redox poise of the endoplasmic reticulum. Free. Radic. Biol. Med. 80, 171-182 (2015).

60. Anelli, T., Sannino, S. \& Sitia, R. Proteostasis and "redoxtasis" in the secretory pathway: tales of tails from ERp44 and immunoglobulins. Free Radic. Biol. Med. 83, 323-330 (2015).

61. Hatahet, F. \& Ruddock, L. W. Modulating proteostasis: peptidomimetic inhibitors and activators of protein folding. Curr. Pharm. Des. 15, 2488-2507 (2009)

62. Oka, O. B., Pringle, M. A., Schopp, I. M., Braakman, I. \& Bulleid, N. J. ERdj5 is the ER reductase that catalyzes the removal of non-native disulfides and correct folding of the LDL receptor. Mol. Cell 50, 793-804 (2013).

63. Ushioda, R. et al. ERdj5 is required as a disulfide reductase for degradation of misfolded proteins in the ER. Science 321, 569-572 (2008).
64. Delaunay-Moisan, A. \& Appenzeller-Herzog, C. The antioxidant machinery of the endoplasmic reticulum: protection and signaling. Free. Radic. Biol. Med. 83, 341-351 (2015)

65. Kakihana, $T$, Nagata, K. \& Sitia, R. Peroxides and peroxidases in the endoplasmic reticulum: integrating redox homeostasis and oxidative folding. Antioxid. Redox. Signal. 16, 763-771 (2012).

66. Zito, E. et al. Oxidative protein folding by an endoplasmic reticulum-localized peroxiredoxin. Mol. Cell 40, 787-797 (2010)

67. Cremers, C. M. \& Jakob, U. Oxidant sensing by reversible disulfide bond formation. J. Biol. Chem. 288, 26489-26496 (2013).

68. Ruddock, L. W. Low-molecular-weight oxidants involved in disulfide bond formation. Antioxid. Redox. Signal. 16, 1129-1138 (2012).

69. Chae, H. J. et al. Bl-1 regulates an apoptosis pathway linked to endoplasmic reticulum stress. Mol. Cell. 15, 355-366 (2004)

70. Eletto, D., Chevet, E., Argon, Y. \& Appenzeller-Herzog, C. Redox controls UPR to control redox. J. Cell Sci. 127(Pt. 17), 3649-3658 (2014).

71. Chakravarthi, S. \& Bulleid, N. J. Glutathione is required to regulate the formation of native disulfide bonds within proteins entering the secretory pathway. J. Biol. Chem. 279, 39872-39879 (2004).

72. Chakravarthi, S., Jessop, C. E. \& Bulleid, N. J. The role of glutathione in disulphide bond formation and endoplasmic-reticulum-generated oxidative stress. EMBO Rep. 7, 271-275 (2006).

73. Molteni, S. N. et al. Glutathione limits Ero1-dependent oxidation in the endoplasmic reticulum. J. Biol. Chem. 279, 32667-32673 (2004).

74. Poet, G. J. et al. Cytosolic thioredoxin reductase 1 is required for correct disulfide formation in the ER. EMBO. J. 36, 693-702 (2017).

75. Bogdanov, A. M. et al. Green fluorescent proteins are light-induced electron donors. Nat. Chem. Biol. 5, 459-461 (2009).

76. Lukyanov, K. A. \& Belousov, V. V. Genetically encoded fluorescent redox sensors. Biochim. Biophys. Acta 1840, 745-756 (2014).

77. Yano, $T$. et al. A novel fluorescent sensor protein for visualization of redox states in the cytoplasm and in peroxisomes. Mol. Cell. Biol. 30, 3758-3766 (2010).

78. D’Autreaux, B. \& Toledano, M. B. ROS as signalling molecules: mechanisms that generate specificity in ROS homeostasis. Nat. Rev. Mol. Cell. Biol. 8 813-824 (2007)

79. Kirstein, J. et al. Proteotoxic stress and ageing triggers the loss of redox homeostasis across cellular compartments. EMBO J. 34, 2334-2349 (2015).

80. Bienert, G. P. et al. Specific aquaporins facilitate the diffusion of hydrogen peroxide across membranes. J. Biol. Chem. 282, 1183-1192 (2007).

81. Medrano-Fernandez, I. et al. Stress regulates aquaporin-8 permeability to impact cell growth and survival. Antioxid. Redox. Signal. 24, 1031-1044 (2016).

82. Bertolotti, M., Bestetti, S., Medrano-Fernandez, I. \& Sitia, R. Response to Marinelli and Marchissio. Antioxid. Redox. Signal. 19, 897 (2013).

83. Bertolotti, M., Farinelli, G., Galli, M., Aiuti, A. \& Sitia, R. AQP8 transports NOX2generated $\mathrm{H}_{2} \mathrm{O}_{2}$ across the plasma membrane to promote signaling in $\mathrm{B}$ cells. J. Leukoc. Biol. 100, 1071-1079 (2016).

84. Marchissio, M. J., Frances, D. E., Carnovale, C. E. \& Marinelli, R. A. Mitochondrial aquaporin-8 knockdown in human hepatoma HepG2 cells causes ROSinduced mitochondrial depolarization and loss of viability. Toxicol. Appl. Pharmacol. 264, 246-254 (2012)

85. Mindthoff, S. et al. Peroxisomal Pex 11 is a pore-forming protein homologous to TRPM channels. Biochim. Biophys. Acta 1863, 271-283 (2016).

86. Antonenkov, V. D., Sormunen, R. T. \& Hiltunen, J. K. The rat liver peroxisomal membrane forms a permeability barrier for cofactors but not for small metabolites in vitro. J. Cell. Sci. 117(Pt. 23), 5633-5642 (2004).

87. Rokka, A. et al. Pxmp2 is a channel-forming protein in Mammalian peroxisomal membrane. PLoS ONE 4, e5090 (2009).

88. Beedle, A. E., Lynham, S. \& Garcia-Manyes, S. Protein S-sulfenylation is a fleeting molecular switch that regulates non-enzymatic oxidative folding. Nat. Commun. 7, 12490 (2016).

89. Finkel, T. From sulfenylation to sulfhydration: what a thiolate needs to tolerate. Sci. Signal. 5, pe10 (2012).

90. Fra, A., Yoboue, E. D. \& Sitia, R. Cysteines as Redox Molecular Switches and Targets of Disease. Front Mol. Neurosci. 10, 167 (2017).

91. Krishnan, N., Fu, C., Pappin, D. J. \& Tonks, N. K. H2S-Induced sulfhydration of the phosphatase PTP1B and its role in the endoplasmic reticulum stress response. Sci. Signal. 4, ra86 (2011)

92. Hourihan, J. M., Moronetti Mazzeo, L. E., Fernandez-Cardenas, L. P. \& Blackwell, T. K. Cysteine sulfenylation directs IRE-1 to activate the SKN-1/Nrf2 antioxidant response. Mol. Cell. 63, 553-566 (2016). 
93. Chouchani, E. T. et al. Mitochondrial ROS regulate thermogenic energy expenditure and sulfenylation of UCP1. Nature 532, 112-116 (2016).

94. Lismont, C. et al. Peroxisomes as modulators of cellular protein thiol oxidation: a new model system. Antioxid. Redox. Signal. https://doi.org/10.1089/ ars.2017.6997 (2017).

95. Peskin, A. V. et al. The high reactivity of peroxiredoxin 2 with $\mathrm{H}(2) \mathrm{O}(2)$ is not reflected in its reaction with other oxidants and thiol reagents. J. Biol. Chem 282, 11885-11892 (2007)

96. Song, I. K. et al. Degradation of redox-sensitive proteins including peroxiredoxins and DJ-1 is promoted by oxidation-induced conformational changes and ubiquitination. Sci. Rep. 6, 34432 (2016).

97. Fourquet, S., Guerois, R., Biard, D. \& Toledano, M. B. Activation of NRF2 by nitrosative agents and $\mathrm{H}_{2} \mathrm{O}_{2}$ involves KEAP1 disulfide formation. J. Biol. Chem. 285, 8463-8471 (2010).

98. Dinkova-Kostova, A. T. et al. Direct evidence that sulfhydryl groups of Keap1 are the sensors regulating induction of phase 2 enzymes that protect against carcinogens and oxidants. Proc. Natl. Acad. Sci. USA 99, 11908-11913 (2002).

99. Wang, J. \& Sevier, C. S. Formation and reversibility of BiP protein cysteine oxidation facilitate cell survival during and post oxidative stress. J. Biol. Chem. 291, 7541-7557 (2016)

100. Petrini, S. et al. Protein glutathionylation in cellular compartments: a constitutive redox signal. Redox. Rep. 17, 63-71 (2012).

101. Ye, Z. W. et al. Glutathione S-tansferase P-mediated protein Sglutathionylation of resident endoplasmic reticulum proteins influences sensitivity to drug-induced unfolded protein response. Antioxid. Redox. Signal. 26, 247-261 (2017)

102. Adachi, T. et al. S-Glutathiolation by peroxynitrite activates SERCA during arterial relaxation by nitric oxide. Nat. Med. 10, 1200-1207 (2004)

103. O'Brien, M., Chalker, J., Slade, L., Gardiner, D. \& Mailloux, R. J. Protein Sglutathionylation alters superoxide/hydrogen peroxide emission from pyruvate dehydrogenase complex. Free. Radic. Biol. Med. 106, 302-314 (2017).

104. Thaher, O. et al. The thiol switch C684 in Mitofusin-2 mediates redox-induced alterations of mitochondrial shape and respiration. Neurochem. Int. pii: S0197-0186(17)30059-1; pii: S0197-0186(17)30059-1. https://doi.org/10.1016/j. neuint.2017.05.009 (2017).

105. Dong, Z. et al. Mitochondrial Ca2+ uniporter Is a mitochondrial luminal redox sensor that augments MCU channel activity. Mol. Cell. 65, 1014-1028 (2017). e1017.

106. Bota, D. A. \& Davies, K. J. Lon protease preferentially degrades oxidized mitochondrial aconitase by an ATP-stimulated mechanism. Nat. Cell. Biol. 4 674-680 (2002)

107. Aksam, E. B. et al. A peroxisomal lon protease and peroxisome degradation by autophagy play key roles in vitality of Hansenula polymorpha cells. Autophagy 3, 96-105 (2007)

108. Omi, S., Nakata, R., Okamura-Ikeda, K., Konishi, H. \& Taniguchi, H. Contribution of peroxisome-specific isoform of Lon protease in sorting PTS1 proteins to peroxisomes. J. Biochem. 143, 649-660 (2008).

109. Bartoszewska, M. et al. Peroxisomal proteostasis involves a Lon family protein that functions as protease and chaperone. J. Biol. Chem. 287, 27380-27395 (2012)

110. Ishii, T. et al. Critical role of sulfenic acid formation of thiols in the inactivation of glyceraldehyde-3-phosphate dehydrogenase by nitric oxide. Biochem. Pharmacol. 58, 133-143 (1999).

111. Yang, Y., Song, Y. \& Loscalzo, J. Regulation of the protein disulfide proteome by mitochondria in mammalian cells. Proc. Natl. Acad. Sci. USA 104, 10813-10817 (2007).

112. Bertolotti, M. et al. Tyrosine kinase signal modulation: a matter of $\mathrm{H}_{2} \mathrm{O}_{2}$ membrane permeability? Antioxid. Redox. Signal. 19, 1447-1451 (2013).

113. Lynes, E. M. \& Simmen, T. Urban planning of the endoplasmic reticulum (ER): how diverse mechanisms segregate the many functions of the ER. Biochim. Biophys. Acta 1813, 1893-1905 (2011).

114. Sitia, R. \& Meldolesi, J. Endoplasmic reticulum: a dynamic patchwork of specialized subregions. Mol. Biol. Cell. 3, 1067-1072 (1992).

115. Lever, J. D. \& Chappell, J. B. Mitochondria isolated from rat brown adipose tissue and liver. J. Biophys. Biochem. Cytol. 4, 287-290 (1958).

116. Shore, G. C. \& Tata, J. R. Two fractions of rough endoplasmic reticulum from rat liver. I. Recovery of rapidly sedimenting endoplasmic reticulum in association with mitochondria. J. Cell. Biol. 72, 714-725 (1977).

117. Rizzuto, R. et al. Close contacts with the endoplasmic reticulum as determinants of mitochondrial Ca2+ responses. Science 280, 1763-1766 (1998)
118. Bilan, D. S. \& Belousov, V. V. HyPer family probes: state of the art. Antioxid. Redox. Signal. 24, 731-751 (2016).

119. Bull, R. et al. Ischemia enhances activation by $\mathrm{Ca} 2+$ and redox modification of ryanodine receptor channels from rat brain cortex. J. Neurosci. 28 9463-9472 (2008)

120. Raturi, A., Ortiz-Sandoval, C. \& Simmen, T. Redox dependence of endoplasmic reticulum (ER) $\mathrm{Ca}(2)(+)$ signaling. Histol. Histopathol. 29, 543-552 (2014).

121. Li, J. H. et al. Calcium overload induces C6 rat glioma cell apoptosis in sonodynamic therapy. Int. J. Radiat. Biol. 87, 1061-1066 (2011).

122. Qin, S., Ding, J., Kurosaki, T. \& Yamamura, H. A deficiency in Syk enhances ceramide-induced apoptosis in DT40 lymphoma B cells. FEBS Lett. 427 139-143 (1998).

123. Lock, J. T., Sinkins, W. G. \& Schilling, W. P. Protein S-glutathionylation enhances $\mathrm{Ca} 2+$-induced $\mathrm{Ca} 2+$ release via the IP3 receptor in cultured aortic endothelial cells. J. Physiol. 590, 3431-3447 (2012).

124. Ushioda, R. et al. Redox-assisted regulation of Ca2+ homeostasis in the endoplasmic reticulum by disulfide reductase ERdj5. Proc. Natl. Acad. Sci. USA 113. E6055-E6063 (2016).

125. Higo, T. et al. Subtype-specific and ER lumenal environment-dependent regulation of inositol 1,4,5-trisphosphate receptor type 1 by ERp44. Cell $\mathbf{1 2 0}$ 85-98 (2005)

126. Raturi, A. et al. TMX1 determines cancer cell metabolism as a thiol-based modulator of ER-mitochondria Ca2+ flux. J. Cell. Biol. 214, 433-444 (2016).

127. Lee, G. H., Lee, H. Y., Li, B., Kim, H. R. \& Chae, H. J. Bax inhibitor-1-mediated inhibition of mitochondrial $\mathrm{Ca} 2+$ intake regulates mitochondrial permeability transition pore opening and cell death. Sci. Rep. 4, 5194 (2014).

128. Lee, G. H. et al. Bax inhibitor-1 regulates endoplasmic reticulum stressassociated reactive oxygen species and heme oxygenase-1 expression. J. Biol. Chem. 282, 21618-21628 (2007).

129. Kim, H. R. et al. Bax inhibitor 1 regulates ER-stress-induced ROS accumulation through the regulation of cytochrome P450 2E1. J. Cell. Sci. 122(Pt 8), 1126-1133 (2009)

130. Orsini, F. et al. The life span determinant p66Shc localizes to mitochondria where it associates with mitochondrial heat shock protein 70 and regulates trans-membrane potential. J. Biol. Chem. 279, 25689-25695 (2004).

131. Lebiedzinska, M., Duszynski, J., Rizzuto, R., Pinton, P. \& Wieckowski, M. R. Agerelated changes in levels of p66Shc and serine 36-phosphorylated p66Shc in organs and mouse tissues. Arch. Biochem. Biophys. 486, 73-80 (2009).

132. Giorgio, M. et al. Electron transfer between cytochrome c andp66(Shc) generates reactive oxygen species that trigger mitochondrial apoptosis. Cell 122, 221-233 (2005).

133. Pinton, $\mathrm{P}$. et al. Protein kinase $\mathrm{C}$ beta and prolyl isomerase 1 regulate mitochondrial effects of the life-span determinant p66Shc. Science $\mathbf{3 1 5}$, 659-663 (2007)

134. Gertz, M., Fischer, F., Wolters, D. \& Steegborn, C. Activation of the lifespan regulator p66Shc through reversible disulfide bond formation. Proc. Natl. Acad. Sci. USA 105, 5705-5709 (2008).

135. Haga, S. et al. Preventing hypoxia/reoxygenation damage to hepatocytes byp66(shc) ablation: up-regulation of anti-oxidant and anti-apoptotic proteins. J. Hepatol. 48, 422-432 (2008).

136. Petrungaro, $\mathrm{C}$. et al. The $\mathrm{Ca}(2+)$-dependent release of the Mia40-induced MICU1-MICU2 dimer from MCU regulates mitochondrial $\mathrm{Ca}(2+)$ uptake. Cell. Metab. 22, 721-733 (2015).

137. Shutt, T., Geoffrion, M., Milne, R. \& McBride, H. M. The intracellular redox state is a core determinant of mitochondrial fusion. EMBO. Rep. 13, 909-915 (2012)

138. Franco, A. et al. Correcting mitochondrial fusion by manipulating mitofusin conformations. Nature 540, 74-79 (2016).

139. Redpath, C. J., Bou Khalil, M., Drozdzal, G., Radisic, M. \& McBride, H. M. Mitochondrial hyperfusion during oxidative stress is coupled to a dysregulation in calcium handling within a C2C12 cell model. PLOS ONE 8, e69165 (2013).

140. Wu, W. et al. FUNDC1 regulates mitochondrial dynamics at the ERmitochondrial contact site under hypoxic conditions. EMBO J. 35, 1368-1384 (2016).

141. Hori, O. et al. Transmission of cell stress from endoplasmic reticulum to mitochondria: enhanced expression of Lon protease. J. Cell. Biol. 157 1151-1160 (2002)

142. Bravo, R. et al. Increased ER-mitochondrial coupling promotes mitochondria respiration and bioenergetics during early phases of ER stress. J. Cell. Sci. 124 (Pt 13)، 2143-2152 (2011). 
143. Yoon, H. et al. Apoptosis induced by manganese on neuronal SK-N-MC cell line: endoplasmic reticulum (ER) stress and mitochondria dysfunction Environ. Health Toxicol. 26, e2011017 (2011)

144. Bhandary, B., Marahatta, A., Kim, H. R. \& Chae, H. J. An involvement of oxidative stress in endoplasmic reticulum stress and its associated diseases. Int. J. Mol. Sci. 14, 434-456 (2012).

145. Leadsham, J. E. et al. Loss of cytochrome c oxidase promotes RAS-dependent ROS production from the ER resident NADPH oxidase, Yno1p, in yeast. Cell. Metab. 18, 279-286 (2013).

146. Liu, Q. et al. A Fenton reaction at the endoplasmic reticulum is involved in the redox control of hypoxia-inducible gene expression. Proc. Natl. Acad. Sci. USA 101, 4302-4307 (2004).

147. Cohen, Y. et al. Peroxisomes are juxtaposed to strategic sites on mitochondria. Mol. Biosyst. 10, 1742-1748 (2014).

148. Mattiazzi Usaj, M. et al. Genome-wide localization study of yeast Pex11 identifies peroxisome-mitochondria interactions through the ERMES complex. J. Mol. Biol. 427, 2072-2087 (2015).

149. Novikoff, P. M. \& Novikoff, A. B. Peroxisomes in absorptive cells of mammalian small intestine. J. Cell. Biol. 53, 532-560 (1972).

150. Kim, P. K., Mullen, R. T., Schumann, U. \& Lippincott-Schwartz, J. The origin and maintenance of mammalian peroxisomes involves a de novo PEX16dependent pathway from the ER. J. Cell. Biol. 173, 521-532 (2006).

151. Fahimi, H. D., Baumgart, E. \& Volkl, A. Ultrastructural aspects of the biogenesis of peroxisomes in rat liver. Biochimie. 75, 201-208 (1993).

152. Zaar, K., Volkl, A. \& Fahimi, H. D. Association of isolated bovine kidney cortex peroxisomes with endoplasmic reticulum. Biochim. Biophys. Acta. 897 135-142 (1987)

153. Dirkx, R. et al. Absence of peroxisomes in mouse hepatocytes causes mitochondrial and ER abnormalities. Hepatology 41, 868-878 (2005).

154. Baumgart, E. et al. Mitochondrial alterations caused by defective peroxisomal biogenesis in a mouse model for Zellweger syndrome (PEX5 knockout mouse). Am. J. Pathol. 159, 1477-1494 (2001)

155. Kovacs, W. J. et al. Peroxisome deficiency causes a complex phenotype because of hepatic SREBP/Insig dysregulation associated with endoplasmic reticulum stress. J. Biol. Chem. 284, 7232-7245 (2009).

156. Yang, T. \& Poovaiah, B. W. Hydrogen peroxide homeostasis: activation of plant catalase by calcium/calmodulin. Proc. Natl. Acad. Sci. USA 99, 4097-4102 (2002).

157. Drago, I., Giacomello, M., Pizzo, P. \& Pozzan, T. Calcium dynamics in the peroxisomal lumen of living cells. J. Biol. Chem. 283, 14384-14390 (2008)

158. Wang, B. et al. Mitochondria are targets for peroxisome-derived oxidative stress in cultured mammalian cells. Free. Radic. Biol. Med. 65, 882-894 (2013)

159. Bienert, G. P. \& Chaumont, F. Aquaporin-facilitated transmembrane diffusion of hydrogen peroxide. Biochim. Biophys. Acta 1840, 1596-1604 (2014).
160. Boveris, A., Oshino, N. \& Chance, B. The cellular production of hydrogen peroxide. Biochem. J. 128, 617-630 (1972).

161. Schrader, M. \& Yoon, Y. Mitochondria and peroxisomes: are the 'big brother' and the 'little sister' closer than assumed? Bioessays 29, 1105-1114 (2007).

162. Antonenkov, V. D. \& Hiltunen, J. K. Transfer of metabolites across the peroxisomal membrane. Biochim. Biophys. Acta 1822, 1374-1386 (2012).

163. Sugiura, A., McLelland, G. L., Fon, E. A. \& McBride, H. M. A new pathway for mitochondrial quality control: mitochondrial-derived vesicles. EMBO J. 33 2142-2156 (2014)

164. van Roermund, C. W., Hettema, E. H., van den Berg, M., Tabak, H. F. \& Wanders, R. J. Molecular characterization of carnitine-dependent transport of acetyl-CoA from peroxisomes to mitochondria in Saccharomyces cerevisiae and identification of a plasma membrane carnitine transporter, Agp2p. EMBO J. 18, 5843-5852 (1999)

165. Delille, H. K., Alves, R. \& Schrader, M. Biogenesis of peroxisomes and mitochondria: linked by division. Histochem. Cell. Biol. 131, 441-446 (2009).

166. Verleur, N., Elgersma, Y., Van Roermund, C. W., Tabak, H. F. \& Wanders, R. J. Cytosolic aspartate aminotransferase encoded by the AAT2 gene is targeted to the peroxisomes in oleate-grown Saccharomyces cerevisiae. Eur. J. Biochem. 247, 972-980 (1997).

167. van Roermund, C. W. et al. Peroxisomal beta-oxidation of polyunsaturated fatty acids in Saccharomyces cerevisiae: isocitrate dehydrogenase provides NADPH for reduction of double bonds at even positions. EMBO J. 17, 677-687 (1998).

168. Hwang, l. et al. Catalase deficiency accelerates diabetic renal injury through peroxisomal dysfunction. Diabetes 61, 728-738 (2012)

169. Koepke, J. I. et al. Restoration of peroxisomal catalase import in a model of human cellular aging. Traffic 8, 1590-1600 (2007)

170. Rucktaschel, R., Halbach, A., Girzalsky, W., Rottensteiner, H. \& Erdmann, R. De novo synthesis of peroxisomes upon mitochondrial targeting of Pex3p. Eur. J. Cell. Biol. 89, 947-954 (2010).

171. Sugiura, A., Mattie, S., Prudent, J. \& McBride, H. M. Newly born peroxisomes are a hybrid of mitochondrial and ER-derived pre-peroxisomes. Nature $\mathbf{5 4 2}$ 251-254 (2017)

172. Filadi, R. et al. Mitofusin 2 ablation increases endoplasmic reticulummitochondria coupling. Proc Natl. Acad. Sci. USA 112, E2174-E2181 (2015).

173. Naon, D. et al. Critical reappraisal confirms that Mitofusin 2 is an endoplasmic reticulum-mitochondria tether. Proc. Natl. Acad. Sci. USA 113, 11249-11254 (2016)

174. Herrera-Cruz, M. S. \& Simmen, T. Cancer: untethering mitochondria from the endoplasmic reticulum? Front Oncol. 7, 105 (2017).

175. Knoblach, B. et al. An ER-peroxisome tether exerts peroxisome population control in yeast. EMBO J. 32, 2439-2453 (2013)

176. Hung, V. et al. Proteomic mapping of cytosol-facing outer mitochondrial and ER membranes in living human cells by proximity biotinylation. Elife $\mathbf{6}$ e24463 (2017) 